\title{
Pharmaceutical Impurities and Degradation Products: An Overview
}

\author{
Prafulla Kumar Sahu1, Suryakanta Swain ${ }^{2 *}$ and Manohar Babu S ${ }^{2}$ \\ ${ }^{1}$ Department of Pharmaceutical Analysis \& Quality Asurance, Raghu College of Pharmacy, Dakamarri, Visakhapatnam-531162, Andhra Pradesh, India \\ ${ }^{2}$ Sims College of Pharmacy, Sims Group of Institutions, Mangaladas Nagar, Vijayawada Road, Guntur-522 001, Andhra Pradesh, India
}

\section{Importance of Open Access and Special Features of Omics Group}

Open access plays a pivotal role in propagating scientific information globally. It is completely user friendly and is open for all to access easily. Its salient features are, that it covers 50 world's leading languages and have the facility of audio versions too. It invites every individual to share as well as explore scientific knowledge and research in the field of pharmaceutical science.

\section{Structure of the Editorial}

Quality, purity, safety and efficacy of pharmaceuticals are the fundamental issues of importance in drug therapy. A greater emphasis is being paid today on assurance of the quality and safety of a drug by monitoring and controlling the impurities and degradation products. The control of pharmaceutical impurities, unwanted substances is a critical issue for the pharmaceutical industry since their presence in the active pharmaceutical constituents and its dosage forms diminish both drug quality and safety. By the identification or characterization and quantification of the impurities and related substances, the risk of their contribution to the side effect profile of the drug therapy can be avoided or minimized. Moreover, the characterization of such trace to minor analytes in the final product is the inherent prerequisites in the wake of stringent requirements from the regulatory authorities. Hence, the development of new analytical methods became an essential task for investigators to determine the stress-related impurities along with their degradation pathway. A forced degradation study (stress testing) is the main tool used to predict stability problems, develop analytical methods, and identify degradation products or pathways. Since, the subject of stress testing has insufficiently addressed in the scientific literature, the measurement, and identification of impurities by today's standards present significant

impurity profiling of drug substances has increasingly viewed as a precious complement to routine investigative work, adding valuable, scientific information in support of their vulnerability to stress conditions and degradation pathways. Impurity profiling has definite techno-commercial value since the pharmaceutical industry is facing the challenge of impurities that diminish the quality, safety and efficacy of drug substances. Due to lack of adequate scientific literature, it is necessary to incorporate stringent tests to control the impurities like by-products, transformation products, degradation products, interaction products, and related products, residual solvents and impurities relating to the inert ingredients (excipients). This fact is also evident from the requirements of the Federal Food, Drug and Cosmetic Act and from a large number of pharmacopeias that provide tests for the control of specific impurities. Forced degradation and impurity profiling is one of the key prerequisites for investigational new drug (IND) and new drug application (NDA) registration. Hence, the estimation of impurity profiles of bulk drug substances is a frequent task, especially in industrial analytical research and quality control laboratories. The significance of impurity profiling has well reflected by the fact that in the recent years numerous books, book chapters, review papers and innumerable ordinary papers have been published on this subject dealing with HPLC, HPTLC, UPLC, LC-MS/MS and many more aspects to emphasize impurity profiling as a hot topic in contemporary pharmaceutical analysis. Impurity profiling study henceforth may be highly supportive for process development, quality assurance as well as routine analysis of commercial formulations. Stress testing and stability indicating analytical procedures provide data to support the characterization of potential degradants; degradation pathways and intrinsic stability of the drug molecule. Unanimously, combinations of chromatographic and spectroscopic techniques have been proved as valuable tools for forced degradation and estimation of impurity profiles in drugs and related materials. Fortunately, the revolutionary advent of a variety of sophisticated hyphenated techniques with liquid chromatography has made this much simpler than the traditional time-consuming approach of quantification, isolation, followed by acquisition of the spectral (mass, UV, NMR and IR) and elemental (C, $\mathrm{H}, \mathrm{N}$ ) data.

On behalf of the editor and editorial board member for this journal, I would like to thanks the OMICS publishing group 5716 Corsa Ave, Suite 110 Westlake, Los Angeles, A 91362-7354, USA for giving me the opportunity for writing an invited editorial article for publication in the upcoming issue.

*Corresponding author: Suryakanta Swain, Associate Professor in Pharmaceutics, SIMS College of Pharmacy, SIMS Group of Institutions, Mangaladas Nagar, Vijayawada Road, Guntur-522 001, Andhra Pradesh, India, Fax: +91-863-2321686; Tel: +91-7702247710/9438038643; E-mail: swain_suryakant@yahoo.co.in

Received January 07, 2015; Accepted January 08, 2015; Published January 10, 2015

Citation: Sahu PK, Swain S, Babu MS (2015) Pharmaceutical Impurities and Degradation Products: An Overview. Pharmaceut Reg Affairs 4: e146. doi: 10.4172/2167-7689.1000e146

Copyright: $\odot 2015$ Sahu PK, et al. This is an open-access article distributed under the terms of the Creative Commons Attribution License, which permits unrestricted use, distribution, and reproduction in any medium, provided the original author and source are credited. 